\title{
Prognostic Value of BCL2 in Women Patients with Invasive Breast Cancer
}

\author{
E-Z Ayadi ${ }^{1,2,3}$, B Cherif ${ }^{1 *}$, Y Ben Hamed ${ }^{4}$, N Ben Yahia $^{5}$, M Mokni ${ }^{3}$, A Rebai ${ }^{1}$, \\ H Ayadi', R Jlidi²
}

\begin{abstract}
Background: Breast cancers are heterogeneous, making it essential to recognize several biomarkers for cancer outcome predictions especially in young women where the classical prediction parameters are not suitable. The goal from this study is to evaluate the impact of B cell lymphoma 2 (BCL2), P53 and Ki-67 proteins expression on survival in young women patients with invasive ductal carcinoma. Patients and methods: Samples and clinical data from 238 patients were collected between 2003 and 2017. They were selected according to 2 criteria: age $\leq 40$ years old and most of them are affected by an Invasive Ductal Carcinoma. We evaluated BCL2, P53 and ki-67 expression by immunochemistry test, and then we assessed correlations of these biomarkers expression with patient's clinicopathological characteristics and survival. Results: Triple negative breast cancer group showed a high frequency among our cohort but we emphasize an almost equitable distribution among all molecular groups. Contrary to other studies which reported that luminal A was correlated with better prognosis, our analysis demonstrated that luminal A is correlated with the Scarff, Bloom and Richardson (SBR) grading 2 or SBR grading 3. To better investigate the prognosis, we analyze three biomarkers known by their impact on physiopathology behavior on breast cancer BCL2, ki-67and P53. BCL2 is the more relevant one, it was correlated with molecular subtypes $(p=0.0012)$ and SBR grading $(p=0.0016)$. BCL2 seems to be the good prognostic biomarker related to survival $(\mathrm{p}=0.004)$ with a protective role among patients when endocrine therapy is not provided and Lymph Node (LN) involvement is positive ( $p=0.021, p=0.000$ respectively). Conclusions: The classical prognostic parameters based mainly on the molecular classification in breast cancer seem insufficient in the case of young women. BCL2 protein expression analysis provides a better prognostic value. BCL2 should be clinically associated in current practice when young women specimens are diagnosticated.
\end{abstract}

Keywords: Breast cancer- BCL2- young women- prognosis

Asian Pac J Cancer Prev, 19 (12), 3557-3564

\section{Introduction}

Breast cancer $(\mathrm{BC})$ represents the most common disease in the world (WHO, 2013). It is the most frequent malignant neoplasm affecting Tunisian female patients with an incidence of 27.1/100,000 inhabitants (Maalej et al., 2004). BC is a heterogeneous disease with molecular subtypes that have biological distinctness and different behavior. This heterogeneity covers epidemiological risk factor, natural histories, biological etiology, clinical outcome, pathological characteristics and response to therapies (Peppercorn et al., 2008). Perou et al., (2000) identified four BC subtypes on the basis of gene-expression profiling of 39 invasive breast tumors and three normal breast specimens.

Molecular subtypes are defined by the immunohistochemical expression of estrogen receptor
(ER), progesterone receptor (PR), and human epidermal growth factor receptor 2 (HER2). Analyses of (ER), (PR) and (HER2) expressions have been routine practice for years. Endocrine therapy is considered for patients with hormone receptor positive (ER + and/or PR + ) tumors. Furthermore, the ER negative (ER-), PR negative (PR-), and HER2 negative (HER2-) tumors, also known as triple-negative phenotype TNBC (ER-/PR-/HER2-) is characterized by expression of cytokeratin 5/6 (CK5/6) and/or the epidermal growth factor receptor (EGFR) (Nielsen et al., 2004 ; Change et al., 2008). For these patients, chemotherapy is the only available treatment. Classification and identification of new important biomarkers may help in prognostication and targeting to treatment to those most likely to benefit (Blows et al., 2010).

Many studies were focused to determine the impact 
of several biomarkers on survival and their inclusion in everyday clinical practice. Since 1979 studies were focused on the important role of the anti-proliferative biomarker P53, as the first tumor suppressor gene described (Gasco et al., 2002; Haldar et al., 1994). Then studies have demonstrated that P53 impact in clinical outcome remains controversial (Yang et al., 2013). While, BCL2 is an anti-apoptotic protein and its role is still also controversial. Some authors reported that BCL2 constitute a strong protein marker in $\mathrm{BC}$ (Kallel-Bayoudh et al., 2011), and is favorable prognostic factor in ER positive (Callagy et al., 2008). Other have shown BCL2 protein and gene expression to be a promising prognostic and predictive marker in human cancers (Von Minckwitz et al., 2008) especially node-negative BC (Ali et al., 2012; Paik et al., 2004).

$\mathrm{BC}$ among young women is known by its significant association with a poor prognosis (Anders et al., 2009) and generally correlated with worse pathological features including higher stage at presentation, grade 3, and estrogen receptor (ER) negative status (Gajdos et al., 2000). The distribution of molecular subtypes among women under 40 years old present less Luminal cancers and more HER2 positive and TNBC tumors than older women (Colleoni et al., 2002). Taking into account the heterogeneity of this disease, we choose to investigate the role of Ki-67, P53 and BCL2 on pathological and survival data in young Tunisian women patients reporting the correlation between biomarkers and each impact on survival.

\section{Materials and Methods}

\section{Study patients}

This is a retrospective cohort of young women with invasive BC. All patients are under 40 years old. They were studied between 2003 and 2017. Clinical features of patients were collected in collaboration with the anatomy pathology laboratories and oncologists. Recorded data provided age at diagnosis, $\mathrm{BC}$ grade determined by Elston-Ellis modification of Scarff-Bloom-Richardson grading system (SBR Grade I, II, III), tumor size ( $<2 \mathrm{~cm}, 2-5 \mathrm{~cm}, \geq 5 \mathrm{~cm}$ ), lymph node (LN) status (positive or negative) and vascular invasion (VI) status (positive or negative). In total, 238 patients from Tunisia accurately from Sfax, Sousse and Djerba regions were selected. Clinical analysis also provided data on whether or/ not the patient had been treated with adjuvant hormonal therapy or adjuvant chemotherapy.

\section{Immunohistochemistry}

Tissues samples from patient cohort were prepared in paraffin-embedded slides for protein investigation. Staining was done continuously and progressively according the specimen reception, for all samples, ER, PR, HER2, Ki-67, P53 and BCL2 were revealed using immunohitochemistry (IHC) tests. All markers were performed with specific antibody [primary antibody anti-human]: HER2 (Novocastra NCL-CB11), ER (Novocastra NCL-ER-6F11), PR (Novocastra NCL-PGR-312), Ki-67 (NovocastraNCL-L-KI-67-MM1),
P5 3 (NovocastraNCL-L-P 53 -DO 7), BCL2 (Novocastra NCL-L-BCL2). Secondary antibodies and streptavidine-HRP (Novocastra) were used for recognized antigen revelation. The enzymatic activation of the chromogen results in a visible reaction product at the antigen site. The specimen may then be counterstained using Hematoxyllin and cover slipped. Under a white light microscope, IHC expression interpretations from samples patients compared to positive controls tissues (using Colonic carcinoma for P53 and Tonsil lymphoma for BCL2 or Ki67 immunostaining) or negative controls (using normal breast showing any immunostained cell) were performed using conventional image analysis software.

\section{Immunostaining scoring}

Experienced pathologist (J.R) who had no knowledge of the patients' clinical status and outcomes evaluated immunohistochemistry (IHC) of ER, PR, HER2, Ki-67, P53 and BCL2 proteins expression. Hormone receptors (ER and PR) were considered positive when more than 1\% of infiltrating tumor cell nuclei were stained (Hammond et al., 2010). Tumors were considered positive for HER2 if immunostaining was scored as $3+$ according to Wolff criteria (Wolff et al., 2007; Wolff et al., 2013) and cancers with HER2 scored as 2+ (indeterminate) were assessed through fluorescent in situ hybridization (FISH) test. Immunostaining of Ki-67, P53 or BCL2 biomarkers was scored on the basis of the percentage of positive tumor cells. Therefore, positive cells were defined as cells with strongly and clearly brown immunostaining compared to controls. The scoring was graded according to the percentage of Ki-67, P53 and BCL2 -positive cells, the following scores were assigned: $0(0 \%), 1(1 \%), 2(2 \%)$ 99 (99\%), $100(100 \%)$.

\section{Breast Cancer subtyping}

Based on ER, PR, HER2 and Ki-67 expression with IHC analysis, we classified samples in to 5 groups characterized by; LA group ER/PR +, HER2- and Ki-67 $<20 \%$, LB like-group ER/PR +, HER2- and Ki- $67 \geq 20 \%$ (Tashima et al., 2015), LB group ER/PR + and HER2+, HER2 group ER/PR- and HER2+ and TNBC group ER/ PR - and HER2-.

\section{Statistical analysis}

Descriptive statistics for clinicopathological features were estimated using simple frequency. Bivariate analysis was performed by assessing the correlation between all the markers and prognosis using chi-square test for binary variables, Pearson-rank correlation for quantitative variables and ANOVA test for quantitative variation among class parameter. Survival analyzes were studied by Kaplan Meier Test. A p-value $<0.05$ is considered as statistically significant. All these tests were done using the SPSS 13.0 software version or R-3.2.3 environment. 


\section{Results}

Clinicopathological characteristics of young women patients

The clinicopathological characteristics are listed in Table 1. Among 238 young women affected with invasive $\mathrm{BC}$, the mean of patients' age at diagnosis is $35 \pm 4.2$ years old ranged from 17-40 years old. Tumor size ranged from $0.8 \mathrm{~cm}$ to $16 \mathrm{~cm}$ with a mean size of $4.24 \mathrm{~cm} \pm 2.7(\mathrm{pT} 2)$ and $6.2 \%$ of patients are affected with inflammatory BC. (Table 1). Informative BC cases about LN involvement and vascular invasion showed $64.1 \%$ and $13.7 \%$ positive cases respectively. Only 9.5\% were grade I BC (SBR classification), and $90.5 \%$ were grade II or III $(43.7 \%$ and $46.8 \%$ respectively).

Based on immunohistochemistry staining of ER, PR and HER2 positive rates were $60.5 \%, 60.1 \%$ and $37.1 \%$

Table 1. Clinical Pathology Characteristics of Young Women Patients with Breast cancer

\begin{tabular}{|c|c|c|c|}
\hline $\begin{array}{l}\text { Clinicopathological } \\
\text { Data }\end{array}$ & Characteristics & Frequency & $\begin{array}{l}\text { Percent } \\
(\%)\end{array}$ \\
\hline Tumor size & $\mathrm{T} 1<2 \mathrm{~cm}$ & 38 & 18.2 \\
\hline \multirow[t]{3}{*}{ Total $=209$} & $2<\mathrm{T} 2<5 \mathrm{~cm}$ & 110 & 52.6 \\
\hline & $\mathrm{T} 3>5 \mathrm{~cm}$ & 48 & 23 \\
\hline & T4 inflammatory & 13 & 6.2 \\
\hline Lymphe Node status & Negative & 69 & 35.9 \\
\hline Total $=192$ & Positive & 123 & 64.1 \\
\hline SBR grading & I & 21 & 9.5 \\
\hline \multirow[t]{2}{*}{ Total $=222$} & II & 97 & 43.7 \\
\hline & III & 104 & 46.8 \\
\hline Vascular invasion & Negative & 88 & 86.3 \\
\hline Total $=102$ & Positive & 14 & 13.7 \\
\hline ER status & Negative & 94 & 39.5 \\
\hline Total $=238$ & Positive & 144 & 60.5 \\
\hline PR status & Negative & 95 & 39.9 \\
\hline Total $=238$ & Positive & 143 & 60.1 \\
\hline HER2 status & Negative & 149 & 62.9 \\
\hline Total $=237$ & Positive & 88 & 37.1 \\
\hline Ki-67 20\% status & Negative & 71 & 46.4 \\
\hline Total $=153$ & Positive & 82 & 53.6 \\
\hline MolecularSubtypes & Luminal A & 38 & 18.72 \\
\hline \multirow[t]{4}{*}{ Total $=203$} & Luminal B Like & 30 & 14.77 \\
\hline & Luminal B & 53 & 26.1 \\
\hline & HER2+ & 35 & 17.24 \\
\hline & TNBC & 47 & 23.15 \\
\hline Overall Survival & $>2$ years & 75 & 54 \\
\hline Total $=139$ & $<2$ Years & 64 & 46 \\
\hline Metastasis & 0 & 106 & 67.9 \\
\hline Total $=156$ & 1 & 50 & 32.1 \\
\hline
\end{tabular}

ER, estrogen receptor; HER2, human epidermal growth factor receptor 2; PR, progesterone receptor;SBR grading, Scarff Bloom and Richardson grading; T, tumor; LA group, luminal A (hormonal receptor $(+)$, her2 (-) and ki-67 <20\%); LB group, luminal B (hormonal receptor $(+)$ and her2 (+)); LB Like group, luminal B (hormonal receptor $(+)$, her2 (-) and ki-67 $\geq 20 \%$ ); HER2 group, human epidermal growth factor receptor 2 (hormonal receptor (-) and her2 (+)); TNBC group, triple negative breast cancer (hormonal receptor (-) and her2 (-)); hormonal receptor $=\mathrm{ER}$ and $\mathrm{PR}$.

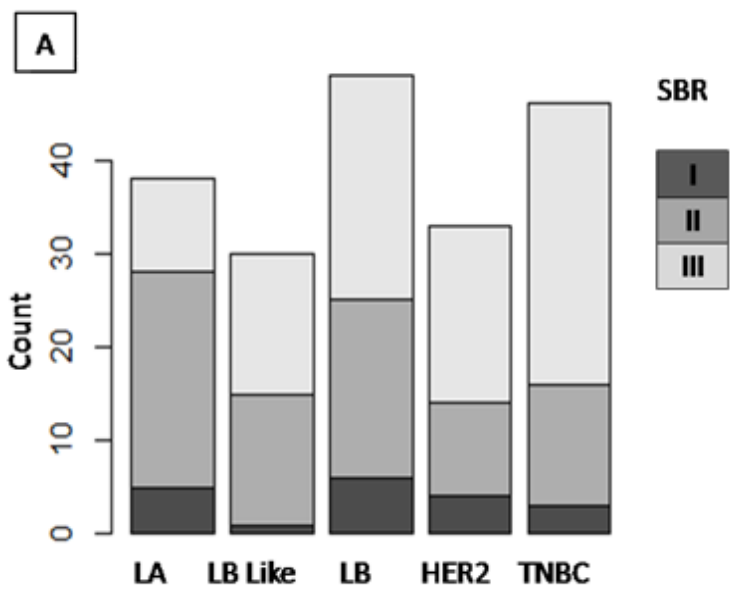

\section{B}

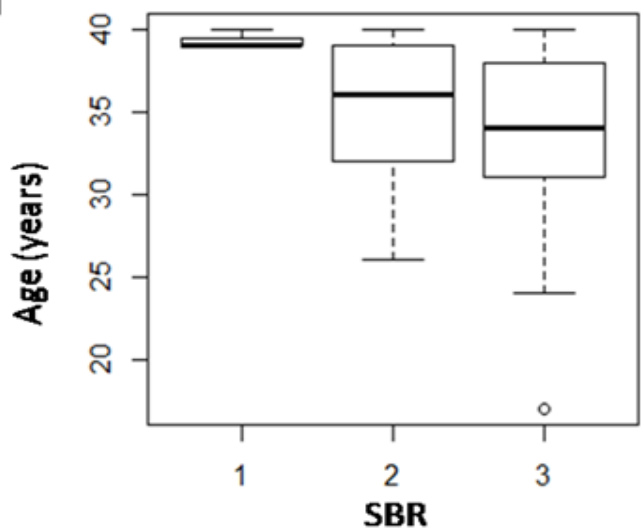

Figure 1. Association between Clinical and Histopathological Parameters of Young Women Breast Cancer Patients. (A) Barplots of SBR grading distribution among molecular subtypes $(p$-value $=0.004)$. (B) Boxplots of patients age according to SBR grading (p-value=0.045). SBR grading, Scarff Bloom and Richardson grading; LA group, luminal A (hormonal receptor $(+)$, her2 (-) and ki-67 $<20 \%$ ); LB group, luminal B (hormonal receptor $(+)$ and her2 (+)); LB Like group, luminal B (hormonal receptor $(+)$, her2 $(-)$ and ki$67 \geq 20 \%$ ); HER2 group, human epidermal growth factor receptor 2 (hormonal receptor (-) and her2 (+)); TNBC group, triple negative breast cancer (hormonal receptor (-) and her2 (-)); hormonal receptor= estrogen receptor and progesterone receptor.

respectively. Ki-67 was assessed in 153 patients. Based on ER, PR, HER2 and Ki-67 rate, samples were classified in to 5 groups as shown in material and methods. Molecular classification showed that Luminal B and TNBC subtypes were the most frequent groups with $26.1 \%$ and $23.15 \%$ respectively followed by LA and HER2 subtypes (Table 1).

\section{Correlation between clinical and histopathological parameters}

Distribution of SBR grading among molecular subtypes showed that $\mathrm{BC}$ with grade I represents only 9.5\% of tumor cases, while SBRI and SBRIII are the most common in studied cohort (Figure 1-A). Statistical analysis showed that there is significant difference of molecular subtype of BC among SBR grading ( $p=0.004)$. These results confirm that $\mathrm{BC}$ in young women presents 


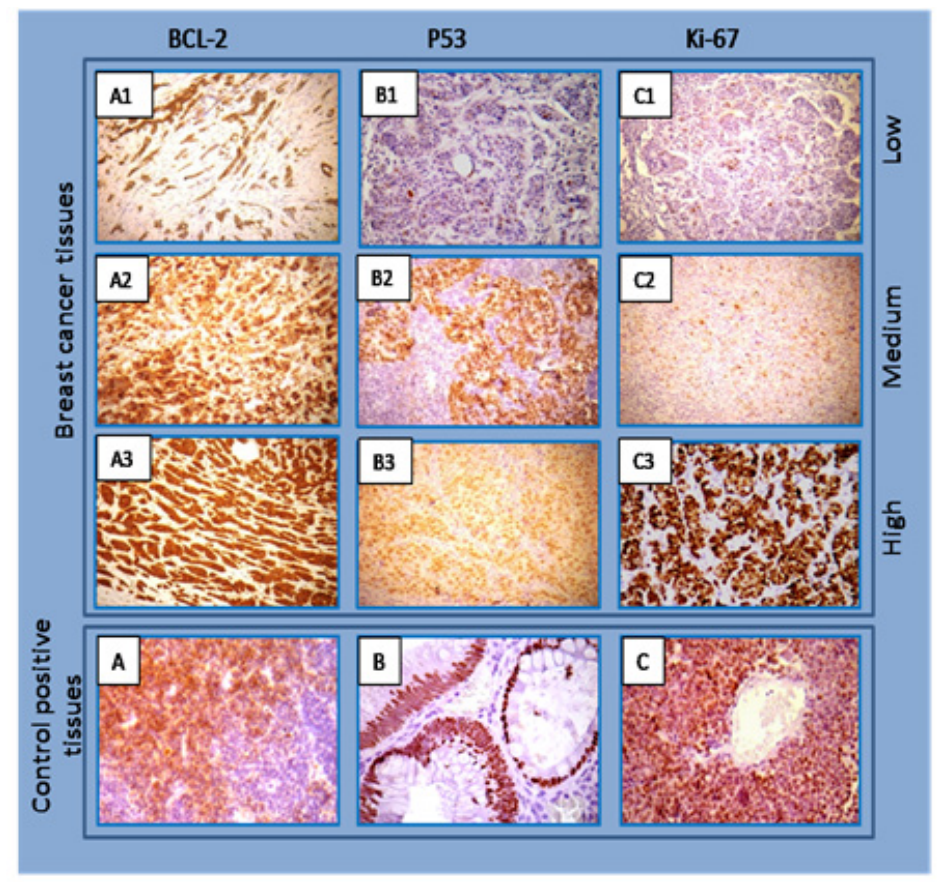

Figure 2. BCL-2, P53 and Ki-67 Expression Analysis by Immunohistochemical Staining in Breast Cancer Tissues. Positive controls revelations are presented by Tonsil lymphoma for BCL2 (A) or Ki67 (C) and Colonic carcinoma for P53 (B) immunostaining. Representative images for low, moderate and high BCL2 (A1-A3), P53 (B1-B3) and Ki-67 (C1-C3) showed biomarkers expression in young women breast cancer specimens. (Original magnification x100).

always an aggressive grade $46.8 \%$ (Table 1 ), therefore HER2+ and TNBC subtypes are the two groups associated with the worst prognosis with SBRIII grading. Moreover, age variation according to the SBR grading shows that the youngest have an SBRIII breast cancers, these data confirm that young age is often associated with the worst grade $(\mathrm{p}<0.05)$ ( Figure 1-B). However, there is no relationship between age of patients and molecular subtypes nor lymph node involvement and vascular invasion.

\section{BCL2, P53 and Ki-67 immunodetection in breast cancer}

BCL2, P53 and Ki-67 expression were evaluated successfully by IHC at least in 140 BC tissues. Based on the extent and intensity of tumor cells, immunostaining was scored as percentage of positive cells according
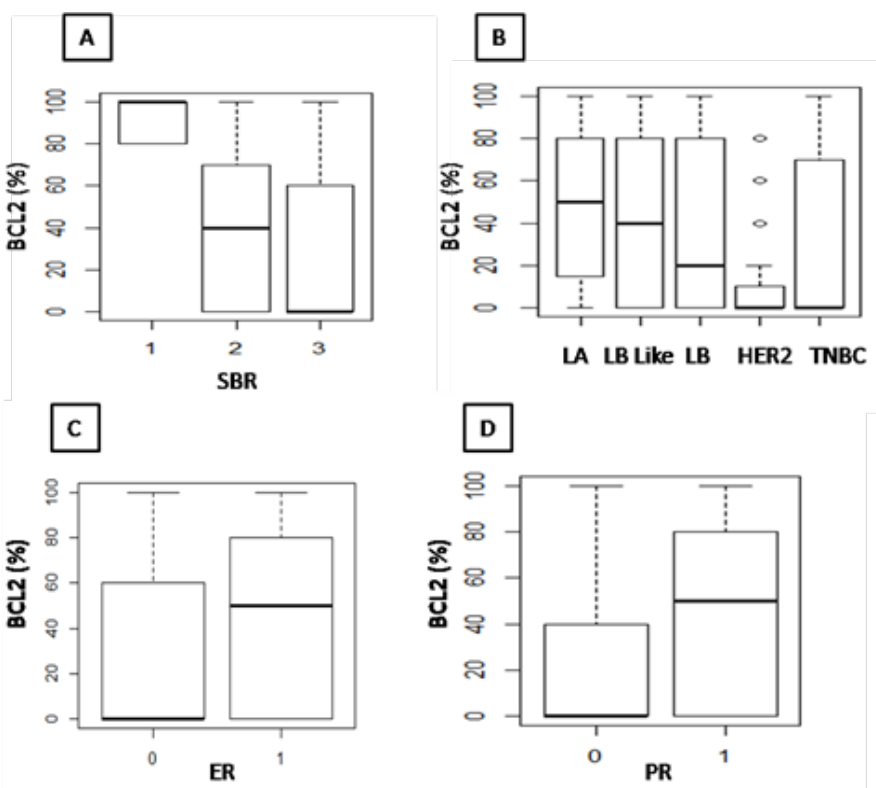

Figure 3. Relationship between BCl-2 Expression and Histopathological Parameters of Young Women Patients with Breast Cancer. Anova tests give significant variation of BCL2 rates among SBR grading (A) ( $p=0.00168)$ showing high percentage with SBR I and molecular groups $(B)(p=0.0012)$ showing high percentage with LA and LB like groups mainly in comparison with HER2 group. Highly correlations were obtained for BCL2 expression levels with estrogen receptor (C) and progesterone receptor (D) ( $\mathrm{p}$-value $<0.001)$. 

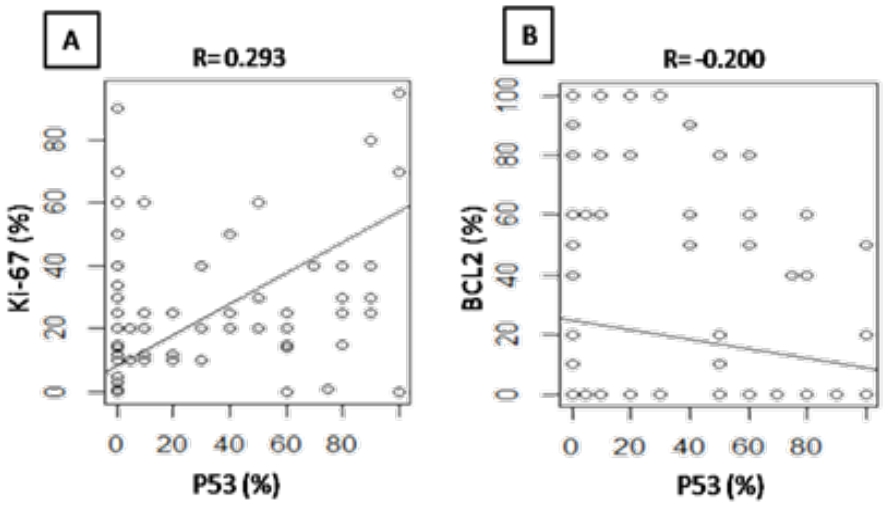

Figure 4. Biomarkers Associations. Plots of P53 levels according to ki-67 levels (A) showing highly significant of positive correlation $(R=0.293, p<0.001)$ but less significant of negative correlation with $B C L-2$ level $(B)(R=-0.200$, $\mathrm{p}<0.05)$. R, coefficient of correlation

to respective biomarker expression localization. An IHC score was generated as described in methods and quantification was performed of cytoplasmic expression for BCL2 (Figure 2, A1-A3) and nuclear expression for P53 (Figure 2, B1-B3) or ki-67 (Figure 2, C1-C3). Choosing this method for scoring is supported by IHC results obtained showing a measurable graduation
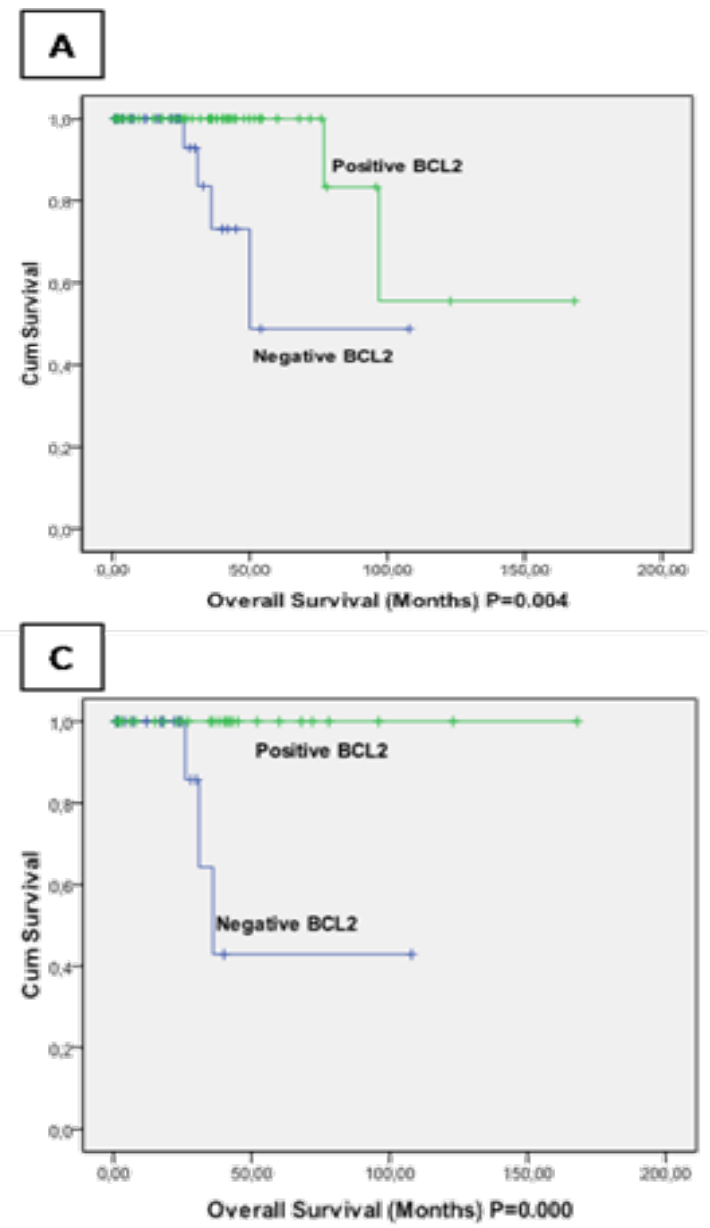

immunostaining revelation, indeed we observe clearly for all biomarkers an expression level ranging from low $(\%$ of positive cells $\leq 30 \%)$, moderate $(30 \leq \%$ of positive cells $\leq 70 \%$ ) to strong ( $\%$ of positive cells $\geq 70 \%$ ) (Figure 2 ). BCL2, P53 and Ki-67 immunodectection are compared and confirmed according to positive controls (Figure 2: A-C) and negative controls (data not shown).
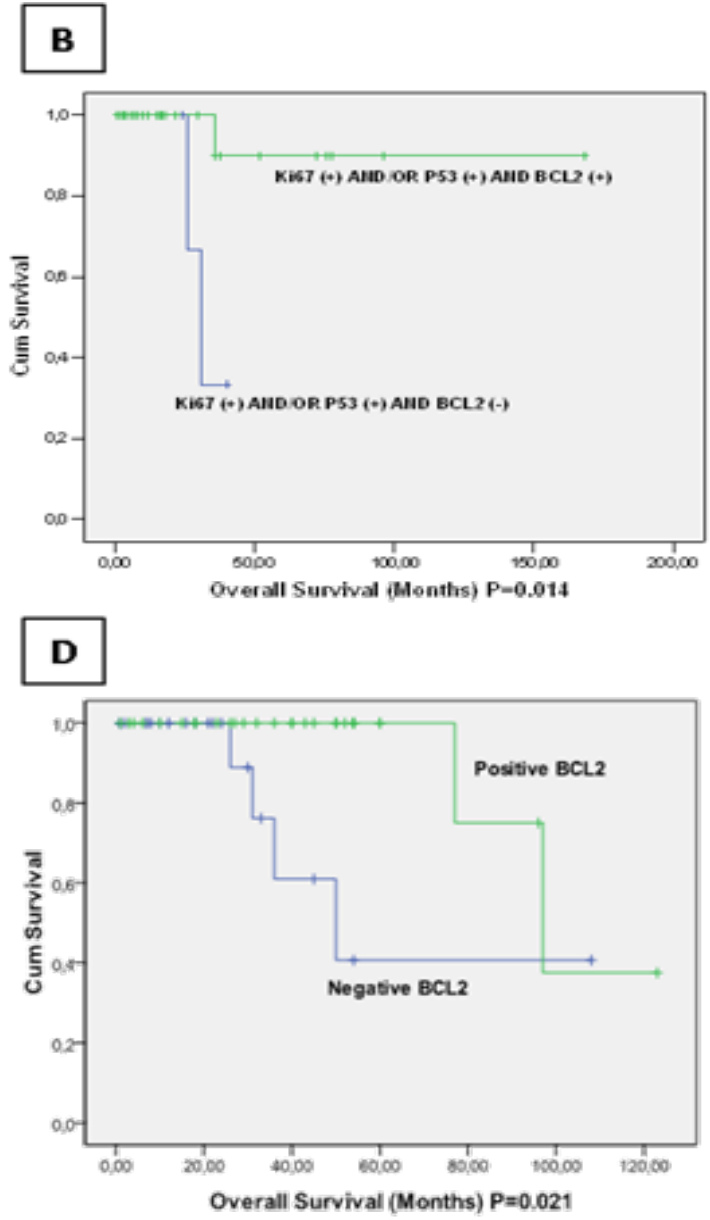

Figure 5. Survival analysis according to BCL2 expression. Graph (A) represents overall survival according to BCL2 expression. Graph (B) shows overall survival according to BCL2 protective impact in presence of Ki-67 or P53. Graph (C) indicates data of overall survival according to BCL2 presence in invasive breast cancer in case of positive node involvement. Graph (D) gives data of overall survival according to BCL2 presence in invasive breast cancer when endocrine therapy is not provided. 


\section{Correlation between all biomarkers and clinical data}

$\mathrm{Ki}-67$ is a marker of cell proliferation, apart from its tissues expression to perform molecular classification, we have investigated its status as a related biomarker among our cohort. Ki-67 expression rate is significantly related to hormonal receptors status $(\mathrm{p}=0.03)$ and $\mathrm{LN}$ involvement $(p=0.045)$. However, Ki-67 levels were slightly progressive with significance limitation according to the SBR grading $(\mathrm{p}=0.068)$ and no significant variations were observed between ki-67 expression and tumor seize. For BCL2 investigations a significant variation of its rate among molecular groups (Figure $3-B, p=0.0012$ ) showing high percentage with LA and LB Like groups mainly in comparison with HER2 group.

In addition, statistical analysis showed highly significant differences between the BC SBR grading according to BCL2 expressions, indeed its rates among SBR grading (Figure $3-A, p=0.00168$ ) showed high percentage in SBR I. Therefore, a similar highly significant correlations were observed between BCL2 levels and ER status (Figure $3-\mathrm{C}, \mathrm{p}=0.000882$ ) and PR status (Figure $3-\mathrm{D}, \mathrm{p}=0.000528)$.

For P53 investigations, surprisingly no significant relationship was observed regarding P53 expression with clinical parameters. However relationship investigations between analyzed biomarkers showed that P53 was positively correlated to Ki-67 (Figure 4A, $\mathrm{R}=0.293$ ) $(\mathrm{p}<0.001)$ and negatively correlated to BCL2 (Figure $4 B, R=-0.200)(p<0.05)$ these suggest that P53 can be indirectly related to a poor prognosis.

\section{Biomarkers and disease outcome}

A significant correlation between BCL2 and overall survival $(\mathrm{p}=0.004)$ (Figure 5-A). These data show that BCL2 is an important biomarker to evaluate prognosis. Patients expressing BCL2 have a better outcome than patients with negative BCL2. However, no significant results were found between survival and $\mathrm{Ki}-67$ or P53 (data not shown).

To better investigate the role of BCL2 and its relation with the two important biomarkers Ki-67 and P53, we had analyzed survival according to BCL2 when one of the two biomarkers Ki-67 and P53 is at least expressed. Figure 5-B indicates that BCL2 is a strong biomarker with a protective effect and that its presence is significantly correlated with a better overall survival even if Ki-67 or/ and P53 are expressed $(\mathrm{p}=0.014)$.

Patients who have positive node involvement have more risk to recurrence, we wanted to explore if BCL2 can better evaluate the prognosis for this group of patients. Figure 5-C, demonstrated that this group of patients has a better outcome of survival if they express BCL2 $(p=0.000)$. Surprisingly when we analyzed the group of patients who didn't receive endocrine therapy and focused on the impact of BCL2, we found that patients who expressed BCL2 have better survival than those who didn't express BCL2 ( $\mathrm{p}=0.021$ ) (Figure 5-D).

\section{Discussion}

Our results showed that $46 \%$ of our patients have a tumor size ranging from 2 to $5 \mathrm{~cm}$ followed by $\mathrm{T} 3>5 \mathrm{~cm}$ with a percentage of 25.7 (Table 1). This is probably due to the rapid proliferation features of $\mathrm{BC}$ in young patients compared to elderly patients (Maggard et al., 2003; Althuis et al., 2009) and that the breast tissue of younger women is denser and therefore it is more difficult to detect $\mathrm{BC}$ by physical examination and mammography (Schreer et al., 2009; Lin et al., 2009).

In our cohort, $\mathrm{BC}$ patients are most likely to present luminal B subtype followed by TNBC. These results disagree with others showing that younger breast cancer patients had a higher prevalence of luminal A and a lower prevalence of basal-like subtype compared with older patients (Tang et al., 2011; Farouk et al., 2016). However, our cohort molecular feature resemble to others who found that LB was the most common type among young women patients (Collins et al., 2012; Fourati et al., 2014). Data disagreement can be explained by differences in genetic backgrounds of populations studied, so comparable results are supported by population's consanguinity through different nations.

This study focuses on the different interactions between BCL2, P53 and Ki-67 in front of clinical data. Significant correlations have be found between Ki-67 and others biomarkers, assuming that $\mathrm{Ki}-67$ is positively correlated with P53, high SBR grading and lymph node involvement. However, we didn't found any significant correlations between Ki-67 or P53 with overall survival (data not shown). Otherwise BCL2 was found positively correlated with ER and PR status and inversely correlated with P53 and high SBR grading $(\mathrm{p}<0.05$ and $\mathrm{p}<0.01$ respectively) (Figure 3 and Figure 4).

Recent studies have shown that BCL2 protein is a promising prognostic and predictive marker in $\mathrm{BC}$, especially in hormone receptor-positive, LN negative BC (Kallel-Bayoudh et al., 2011; Ali et al., 2012; Paik et al., 2004). BCL2 protein expression in tumors was first studied in non-Hodgkin's lymphoma (Yang et al., 2013; Chan et al., 2011; Chin et al., 2005; Horwitz and Alpha-crystallin, 2003; Lambi et al., 2011). Many studies have demonstrated that BCL2 is a strong prognostic factor correlated with a better survival (Martinez-Arribas et al., 2007; Dawson et al., 2010; Callagy et al., 2006). Our results confirm this findings, we proved that $\mathrm{BC}$ young women expressing BCL2 have a better Overall Survival $(\mathrm{p}=0.004)$ (Figure 5-A).

Moreover, it has been reported that $\mathrm{Ki}-67$ is an important biomarker which provides additional and independent predictive information regarding the response to chemotherapy and the prognosis in a group of patients receiving neoadjuvant treatment for BC (Fasching et al., 2011). So, this marker can be used to select patients who are unable to benefit from chemotherapy, such as those with HER2-negative and hormone receptor-positive tumors with low proliferation (Fasching et al., 2011; Inwald et al., 2013). In despite of some authors who have found that P53 protein was associated with high tumor proliferation rate, early disease recurrence and early death in node negative BC (Allred et al., 1993; Yamamoto et al., 2014). Fountzials and colleagues (2016) have proved that HER2 positive and P53 IHC positive 
tumors were associated with increased risk for relapse in the pre-trastuzumab era, while the same phenotype conferred favorable disease free survival (DFS) in the post-trastuzumab era trials.

Keeping in mind these facts, we wanted to focus on supplementary effect that BCL2 can provide in the presence of the 2 prognostic biomarkers Ki-67 and P53. So we devised our patients on two groups; first one expresses at least one of the poor biomarkers and do not express BCL2 and the second one expresses at least one of the poor biomarkers and express BCL2. We analyzed survival in these two groups using Kaplan Meier test. Knowing that high Ki-67/BCL2 index is correlated with short disease free survival (Min et al., 2016). Our result confirmed that BCL2 do have a supplementary positive effect on survival with protective role $(\mathrm{p}=0.014)$ (Figure 5-B). Raising this result, we want to know better about this protective role. For this purpose, we studied the protective role of BCL2 among patients with positive node involvement and patients with endocrine therapy not provided. In our cohort $64 \%$ are node positive, this criterion is known to be associated with high risk of recurrence and bad survival (Kim et al., 2016), (Table 1). Hence, the protective role of BCL2 was confirmed inside the two groups presenting a better survival correlated with the presence of BCL2 $(p=0.000, p=0.021$ respectively) (Figure 5-C, D).

In conclusion, different molecular subtypes will lead to different prognosis and therapeutic option. Thus, molecular subtyping is essential for breast carcinoma management. But considering only the molecular group to establish a prognostic value is insufficient in young woman where the physiopathology seems to be different from their counterpart. Our results revealed that BCL2 is the best prognostic biomarker with a protective role. BCL2 expression analysis seems to be essential in medical practice to better evaluate $\mathrm{BC}$ prognosis in young women.

\section{Abbreviations}

ANOVA, analysis of variance; BCL2, B cell lymphoma 2; BC, Breast cancer; ER, estrogen receptor; HER2, growth factor receptor 2; LA, luminal A; LB, luminal B; LN, lymph node; PR, progesterone receptor; SBR grade (I, II, III), Scarff-Bloom-Richardson grading system; TNBC, triple negative breast cancer; VI, vascular invasion.

\section{Formating of funding sources}

This study was funded by ISESCO (Islamic Educational, Scientific and Cultural Organization) Research grant (Ref N²148) and Bioprotech/Centre of Biotechnology of Sfax grant $\left(\operatorname{Ref} \mathrm{N}^{\circ} 26625\right)$.

\section{Conflict of Interest}

Boutheina Cherif has received research grants from ISECO and Hammadi Ayadi has received research grants from Era Wide UE FP7 Bioprotech Project (Ref ${ }^{\circ}{ }^{26625}$ ).

\section{Acknowledgements}

The authors would like to thank all participants for their contribution and cooperation. This work was partially supported by ISESCO (Islamic Educational, Scientific and Cultural Organization) Research grant (Ref $\left.N^{\circ} 2148\right)$ and Era Wide UE FP7 Bioprotech Project (Ref N²6625).

\section{References}

Ali HR, Dawson SJ, Blows FM E, et al (2012). A KI-67/BCL2 index based on immunohistochemistry is highly prognostic in ER-positive breast cancer. $J$ Pathol, 226, 97-107.

Allred DC, Clark GM, Elledge R, et al (1993). Association of P53 protein expression with tumor cell proliferationrate and clinical outcome in node-negative breast cancer. $J$ Natl Cancer Inst, 85, 200-6.

Althuis MD, Brogan DD, Coates RJ, et al (2003). Breast cancers among very young premenopausal women (United States). Cancer Causes Control, 14, 151-60.

Anders CK, Johnson R, Litton J, Phillips M, Bleyer A (2009). Breast cancer before age 40 years. Semin Oncol, 36, 237-49.

Blows FM, Driver KE, Schmidt MK, et al (2010). Subtyping of breast cancer by immunohistochemistry to investigate a relationship between subtype and short and long term survival: a collaborative analysis of data for 10,159 cases from 12 studies. PLoS Med, 7, e1000279.

Callagy GM, Webber MJ, Pharoah PD, Caldas C (2008). Meta-analysis confirms BCL2 is an independent prognostic marker in breast cancer. BMC Cancer, 29, 153.

Callagy GM, Pharoah PD, Pinder SE, et al (2006). BCL2 is a prognostic marker in breast cancer independently of the Nottingham Prognostic Index. Clin Cancer Res, 12, 2468-75.

Chan S, Lui PC, Tan P, et al (2011). Increased alpha-Bcrystallin expression in mammary metaplastic carcinomas. Histopathology, 59, 247-55.

Change MC, Voduc D, Bajdik C, et al (2008). Basal-like breast cancer defined by five biomarkers has superior prognostic value than triple-negative phenotype. Clin Cancer Res, 14, 1368-76.

Chin D, Boyle GM, Williams RM, et al (2005). Alpha B-Crystallin, a new independent marker for poor prognosis in head and neck cancer. Laryngoscope, 115, 1239-42.

Colleoni M, Rotmensz N, Robertson C, et al (2002). Very young women ( $<35$ years) with operable breast cancer: features of disease at presentation. Ann Oncol, 13, 273-9.

Collins LC, Marotti JD, Gelber S, et al (2012). Pathologic features and molecular phenotype by patient age in a large cohort of young women with breast cancer. Breast Cancer Res Treat, 131, 1061-6.

Dawson S, Makretsov N, Blows F, et al (2010). BCL2 in breast can-cer: a favourable prognostic marker across molecular subtypes and independent of adju-vant therapy received. Br J Cancer, 103, 668-75.

Farouk O, Ebrahim MA, Senbel A, et al (2016). Breast cancer characteristics in very young Egyptian women $\leq 35$ years. Breast Cancer, 5, 53-8.

Fasching PA, Heusinger K, Haeberle L, et al (2011). KI-67, chemotherapy response, and prognosisin breast cancer patients receiving neoadjuvant treatment. BMC Cancer, 14, 486

Fountzilas G, Giannoulatou E, Alexopoulou Z, et al (2016). TP53 mutations and protein immunopositivity may predict for poor outcome but also for trastuzumab benefit in patients with early breast cancer treated in the adjuvant setting. Oncotarget, 22, 32731-53.

Fourati A, Boussen H, El May MV, et al (2014). Descriptive analysis of molecular subtypes in Tunisian breast cancer. Asia Pac J Clin Oncol, 10, 69-4.

Gajdos C, Tartter PI, Bleiweiss IJ, Bodian C, Brower ST (2000). Stage 0 to stage III breast cancer in young women. $\mathrm{J} \mathrm{Am}$ Coll Surg, 190, 523-9.

Asian Pacific Journal of Cancer Prevention, Vol $19 \mathbf{3 5 6 3}$ 
Gasco M, Shami S, Crook T (2002). The P53 pathway in breast cancer. Breast Cancer Res, 4, 70-6.

Haldar S, Negrini M, Monne M, Sabbioni S, Croce CM (1994). Down-regulation of BCL2 by P53 in breast cancer cells. Cancer Res, 54, 2095-7.

Hammond ME, Hayes DF, Dowsett M, et al (2010). American Society of Clinical Oncology; College of American Pathologists. American Society of Clinical Oncology/College of American Pathologists guideline recommendations for immunohistochemical testing of estrogen and progesterone receptors in breast cancer [unabridged version]. Arch Pathol Lab Med, 134, 48-72.

Horwitz J (2003). Alpha-crystallin. Exp Eye Res, 76, 145-3.

Inwald EC, Klinkhammer-Schalke M, Hofstädter ,F et al (2013). KI-67 is a prognostic parameter in breast cancerpatients: results of a large population-based cohort of a cancer registry. Breast Cancer Res Treat, 139, 539-2.

Kallel-Bayoudh I, Hassen HB, Khabir A, et al (2011). BCL2 expression and triple negative profile in breast carcinoma. Med Oncol, 28, 55-61.

Kim SW, Chun M, Han S, et al (2016). Young age is associated with increased locoregional recurrence in node-positive breast cancer with luminal subtypes. Cancer Res Treat, 49, 484-3.

Lambi F, Moldoveanu T, Tait SW, et al (2011). A unified model of mammalian BCL2 protein family interactions at the mito-chondria. Mol Cell, 44, 517-31.

Lin CH, Liau JY, Lu YS et al (2009). Molecular subtypes of breast cancer emerging in young women in Taiwan: evidence for more than just westernization as a reason for the disease in Asia. Cancer Epidemiol Biomarkers Prev, 18, 1807-14.

Maalej M, Hentati D, Messai T, et al (2008). Breast cancer in Tunisia in 2004: a comparative clinical and epidemiological study. Bull Cancer, 95, 5-9.

Maggard MA, O'Connell JB, Lane KE et al (2003). Do young breast cancer patients have worse outcomes?. J Surg Res, 113, 109-13.

Martinez-Arribas F, Alvarez T, Del Val G, et al (2007). BCL2 expression in breast cancer: a comparative study at the mRNA and protein level. Anticancer Res, 27, 219-22.

Min KW, Kim DH, Do SI, et al (2016). High KI-67/BCL2 index is associated with worse outcome in early stage breast cancer. Postgrad Med J, 92, 707-14.

Nielsen TO, Hsu FD, Jensen K, et al (2004). Immunohistochemical and clinical characterization of the basal-like subtype of invasive breast carcinoma. Clin Cancer Res, 10, 5367-74.

Paik S,Shak S, Tang G, et al (2004). A multigene assay to predict recurrence of tamoxifen-treated, node-negative breast cancer. N Engl J Med, 351, 2817-26.

Peppercorn J, Perou CM, Carey LA (2008). Molecular subtypes in breast cancerevaluation and management: divide and conquer. Cancer Invest, 26, 1-10.

Perou CM, Sørlie T, Eisen MB, et al (2000). Molecular portraits of human breast tumours. Nature, 406, 747-52.

Schreer I (2009). Dense breast tissue as an important risk factor for breast cancer and implications for early detection. Breast Care, 4, 89-92.

Tang J, Wu CC, Xie ZM, Luo RZ, Yang MT (2011). Comparison of clinical features and treatment outcome of breast cancers in young and elderly Chinese patients. Breast Care [Basel], 6, 435-40.

Tashima R, Nishimura R, Osako T, et al (2015). Evaluation of an optimal cut-off point for the KI-67 index as a prognostic factor in primary breast cancer: A retrospective study. PLoS One, 10, e0119565.

Von Minckwitz G, Sinn HP, Raab G, et al (2008). Clinical response after two cycles compared to HER2, KI-67, P53, and BCL2 in independently predicting a pathological complete response after preoperative chemotherapy in patients with operable carcinoma of the breast. Breast Cancer Res, 10, R30.

Wolff AC, Hammond ME, Hicks DG, et al (2013). American Society of Clinical Oncology; College of American Pathologists. Recommendations for human epidermal growth factor receptor 2 testing in breast cancer: American Society of Clinical Oncology/College of American Pathologists clinical practice guideline update. J Clin Oncol, 31, 3997-4013.

Wolff AC, Hammond ME, Schwartz JN, et al (2007). American Society of Clinical Oncology/College of American Pathologists guideline recommendations for human epidermal growth factor receptor 2 testing in breast cancer. Arch Pathol Lab Med, 131, 18-43.

World Health Organization (2013). Breast cancer prevention and control.

Yamamoto M, Hosoda M, Nakano K, et al (2014). P53 accumulation is a strong predictor of recurrence in estrogen receptor-positive breast cancer patients treated with aromatase inhibitors. Cancer Sci, 105, 81-8.

Yang D, Chen M, Wang L, et al (5013). BCL2 expression predicts sensitivity to che-motherapy in breast cancer: a systematic re-view and meta-analysis. J Exp Clin Cancer Res, 32, 105.

Yang P, Du CW, Kwan M, Liang SX, Zhang GJ (2013). The impact of P53 in predictingclinical outcome of breast cancer patients with visceral metastasis. Sci Rep, 3, 2246.

\section{(ब) ब(8)}

This work is licensed under a Creative Commons AttributionNon Commercial 4.0 International License. 